\title{
Tupí-Guaraní e Mundurukú: evidências lexicais e fonológicas de parentesco genético
}

\author{
Aryon Dall'Igna Rodrigues
}

O objetivo desta comunicação é apresentar cerca de 140 pares de cognatos determinados entre as línguas Tupinambá, da família Tupí-Guaraní, e Mundurukú e Kuruáya, da família Mundurukú, e as correspondências fonológicas estabelecidas com base nos mesmos. A família Tupí-Guaraní, embora abrangendo mais de vinte línguas é muito homogênea (cf. Lemle, 1971) e o Tupinambá pode ser tomado como uma língua típica dela. Os dados do Tupinambá provêm de fontes quinhentistas e seiscentistas (especialmente Anônimo, 1952-1953) e foram fonemizados segundo Rodrigues (1958). A família Mundurukú é constituída apenas pelas línguas Mundurukú e Kuruáya (Rodrigues, 1970). Os dados do Mundurukú provêm de Crofts (1973 e comunicação pessoal), suplementados por Strömer (1932). Os dados do Kuruáya são os colhidos e publicados por Nimuendajú (1930). Ambas as famílias são consideradas membros do tronco Tupí (Rodrigues, 1970), mas esta é a primeira vez que se apresentam evidências mais substanciais e sistemáticas de seu parentesco. Um pequeno número de correspondências sonoras foi publicado num estudo classificatório preliminar (Rodrigues, 1955). O primeiro estudo sobre o parentesco Mundurukú-Tupí se deve a Nimuendajú (1937).

$\mathrm{Na}$ lista de cognatos, às formas do Tupinambá (Tb) acrescentam-se, quando possível, as formas reconstruídas para o Proto-Tupí-Guaraní (marcadas com *), em parte conforme a publicação feita por Lemle (1971), em parte inéditas. A essas seguem-se as formas do Mundurukú $(\mathrm{Mu})$ e do Kuruáya $(\mathrm{Ku})$.

As consoantes do Tupinambá são $p t k ? m n \eta s b$ (fricativa bilabial em posições inicial e medial, oclusiva bilabial em posição final) $r w y$; suas vogais são i e i a u o, e as correspondentes nasais. As consoantes do Mundurukú são $p t \check{c} k ? b d \check{J} m n \eta s \check{s} h r w y$; as vogais são $i$ e $\dot{t} a o$, e as correspondentes nasais. As consoantes do Kuruáya são as mesmas do Mundurukú, mais [ $t]$, que corresponde à parte dos $/ r /$ do Mundurukú. As vogais provavelmente são as 
mesmas também, mas, à falta de uma análise fonêmica, mantêm-se nos dados

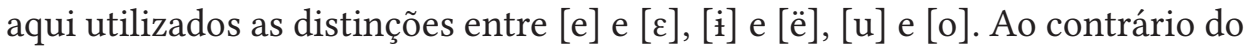
Tupinambá e das línguas Tupí-Guaraní em geral, o Mundurukú é tonal, com pelo menos três níveis de tom (Braun e Crofts, 1965). No presente trabalho comparativo não serão considerados os tons do Mundurukú.

A gramática superficial do Tupinambá difere em muitos respeitos da gramática do Mundurukú. Entretanto, podem ser apontadas semelhanças em parte dos morfemas de referência pessoal (cf. cognatos 36, 37, 38, 39, 83, 84, $130,131,139)$, nos morfemas transitivadores (causativos) e intransitivadores (reflexivo) $(17,18,117)$, em parte dos afixos casuais $(29,40)$ e partículas relacionais $(25,30,41)$, nos nominalizadores $(81,82,86)$.

A seguir são enumeradas as correspondências fonológicas detectadas. Ao lado do Tupinambá $(\mathrm{Tb})$ acrescenta-se o fonema correspondente reconstruído para o Proto-Tupí-Guaraní (*). Ao Mundurukú (Mu) acrescenta-se o Kuruáya $(\mathrm{Ku})$ sempre que possível; note-se, porém, que não há análise fonêmica disponível para o Kuruáya. Sob um mesmo número reúnem-se correspondências que se complementam e que provavelmente são atribuíveis a um só fonema da protolíngua em contextos fonológicos diferentes. Os números dos exemplos remetem à lista de cognatos.

1. $\mathrm{Tb} p,{ }^{*} p=\mathrm{Mu} \emptyset, \mathrm{Ku} \emptyset$ em início de morfema, diante vogal, anterior: 14 , $27,58,92,95,139,140$.

$\mathrm{Tb} p,{ }^{*} p=\mathrm{Mu} b, \mathrm{Ku} b$ em outros ambientes: $16,22,40,47,64,73,80,86$, 89, 94, 102, 113, 123, 126.

2. Tb $p,{ }^{*} p=\mathrm{Mu} p, \mathrm{ku} p, b: 3,6,9,28,35,48,85,99,100,109,119,129,141$ (v. nota 1$)$.

3. Tb $b,{ }^{*} b=\mathrm{Mu} p$, Ku $p,-b: 10,45,46,50,53,60,65,66,75,81,87,90,97,98$, 101, 110, 125, 137, 138, 141.

4. Tb $b,{ }^{*} b=\mathrm{Mu} b, \mathrm{Ku} b: 6,29,108,135$ (v. nota 2).

5. Tb $t,{ }^{*} t=\mathrm{Mu} t, \mathrm{Ku} t: 51,59,78,93,126$.

6. Tb $t,{ }^{*} t=\mathrm{Mu} d, \mathrm{Ku} t-,-d-: 2,55,57,75,119,135,140$ (v. nota 3).

7. $\mathrm{Tb} t,{ }^{*} t=\mathrm{Mu} n, \mathrm{Ku} n$ em sílaba contendo nasal: 12 .

8. $\mathrm{Tb} t,{ }^{*} t=\mathrm{Mu} \check{c}, \mathrm{Ku} t$ em outros ambientes: 132 (v. nota 4$)$. 
Tb $r,{ }^{*} r=\mathrm{Mu} t, \mathrm{Ku} d$ diante de pausa: $13,27,31,34,49,51,62,64,65,66$, 76, 82, 86, 91, 94, 99, 104.

Tb $r,{ }^{*} r=\mathrm{Mu} r$, Ku $r$ em outros ambientes: 9, 88, 102, (v. nota 5).

9. Tb $s,{ }^{*} c=\mathrm{Mu} \check{s}, \mathrm{Ku} \breve{j}$ diante de $\mathrm{Mu} i, \mathrm{Ku} i: 22,71,72,91,95,100,134$.

$\mathrm{Tb} s,{ }^{*} c=\mathrm{Mu} \breve{j}, \mathrm{Ku} d$ em outros ambientes: 21, 24, 25, 41, 63, 111, 121, 128 , 137.

10. $\mathrm{Tb} k,{ }^{*} k=\mathrm{Mu} \check{s}, \mathrm{Ku} \check{s}$ diante de vogal anterior da protolíngua: 8, 34, 60 .

$\mathrm{Tb} k,{ }^{*} k=\mathrm{Mu} k$, Ku $k$ em outros ambientes: 5, 7, 11, 15, 20, 28, 35, 42, 55, $59,62,64,65,67,68,70,74,95,96,101,107,120,123,127,128,132$ (v. nota 6).

11. $\mathrm{Tb} k w,{ }^{*} k^{w}=\mathrm{Mu} k, \mathrm{Ku} k: 90,124$.

12. $\mathrm{Tb}{ }^{\prime},{ }^{*}=\mathrm{Mu} \emptyset, \mathrm{Ku} \emptyset$ intervocálico, contíguo a vogal alta: 28, 48, 50, 64, 85, 89, 103, 106.

$\mathrm{Tb}$ ', * $=\mathrm{Mu}$ ', (Ku?) em outros ambientes: 10, 13, 26, 33, 49, 55, 56, 77, 79, 105, 127 (v. nota 7).

13. Tb $y,{ }^{*} y=\mathrm{Mu}$ ǰno início de morfema: $39,115,136,138$.

$\operatorname{Tb} y,{ }^{*} y=\mathrm{Mu} \emptyset$ no fim de morfema, após proto $-\dot{t}: 80,100$.

$\mathrm{Tb} y,{ }^{*} y=\mathrm{Mu} y$, Ku $y$ no fim de morfema, após outras vogais: 3, 20, 23, 24, 32, 43, 54, 69, 111,112, 126.

14. Tb $y,{ }^{*} y=\mathrm{Mu} w, \mathrm{Ku} w: 11,31,43,54,67,71,76,84,88,93,104,105,117$ (v. nota 8).

15. $\mathrm{Tb} w,{ }^{*} w=\mathrm{Mu} \emptyset: 9,21,112,122(\mathrm{v}$. nota 9).

16. Tb $u,{ }^{*} u=\mathrm{Mu} o, \mathrm{Ku} u, o: 12,26,28,35,45,50,59,67,85,87,88,103,112$, $119,122,128,132,133,135,136,137$ (v. nota 10).

17. $\mathrm{Tb} \tilde{u},{ }^{*} \tilde{u}=\mathrm{Mu} \tilde{o}, \mathrm{Ku} \tilde{u}: 70,78$ (v. nota 10$)$.

18. Tb $\dot{i},{ }^{*} \dot{i}=\mathrm{Mu} i$, Ku $i: 1,10,11,22,27,49,51,62,64,65,66,68,71,72,75$, 78, 86, 91, 92, 94, 95, 100, 101, 106, 109, 119, 122, 123, 125, 134, 135, 138, 140 (v. nota 11$)$.

19. $\mathrm{Tb} i,{ }^{*} i=\mathrm{Mu} i, \mathrm{Ku} i: 28,30,36,57,76,89,93,116$ (v. nota 12). 
20. $\mathrm{Tb} o,{ }^{*} o=\mathrm{Mu} \dot{i}, \mathrm{Ku} \dot{i}, \ddot{e}$ em sílaba acentuada: 3, 5, 6, 7, 15, 17, 18, 22, 23, 24, 47, 53, 55, 61, 63, 73, 94, 113, 120, 126, 127.

$\mathrm{Tb} o,{ }^{*} o=\mathrm{Mu} o, \mathrm{Ku} u$ em sílaba átona: 37, 39, 46, 83, 99, 100 (v. nota 13).

21. Tb $\tilde{o},{ }^{*} \tilde{o}=\mathrm{Mu} \tilde{t}: 20,77$.

22. $\mathrm{Tb} a,{ }^{*} a=\mathrm{Mu} \dot{i}, \mathrm{Ku} a$ diante de consoante nasal final de morfema: 4, 96.

$\mathrm{Tb} a,{ }^{*} a=\mathrm{Mu} a, \mathrm{Ku} a$ em outros ambientes: 2, 9, 13, 19, 21, 24, 31, 43, 46, $48,52,55,56,67,71,74,80,81,82,85,88,89,90,93,97,98,99,102,104,105$, $106,107,110,112,113,115,118,123,124,128$ (v. nota 14).

23. $\mathrm{Tb} \tilde{a},{ }^{*} \tilde{a}=\mathrm{Mu} \tilde{t}, \mathrm{Ku} \tilde{a}: 32,54$.

24. Tb $e,{ }^{*} e=\mathrm{Mu} e$, Ku $e, \varepsilon: 5,8,11,14,16,21,25,29,33,34,40,41,58,76,83$, $114,117,121,130,131,138,139$.

$\mathrm{Tb} e,{ }^{*} e=\mathrm{Mu} \dot{t}$ diante de $-y: 69,111$ (v. nota 15$)$.

25. Tb $V,{ }^{*} V=\mathrm{Mu} \tilde{V}$ diante de consoante nasal final de morfema: 4, 12, 21, 42 , $61,79,96,103,121,131,132$ (v. nota 16).

\section{Lista de cognatos}

1- água: $\mathrm{Tb}-\dot{t},{ }^{*} \dot{t}, \mathrm{Mu}-i$.

2- aldeia: $\mathrm{Tb} t a b,{ }^{*} t a b, \mathrm{Mu}$ da-at abrigo temporário.

3- alimentar: $\mathrm{Tb}$ poy, Mu piy-bit comida, Ku buy-comida.

4- amarrar: Tb man, Mu mĩn.

5- andar, estar: Tb eko, *eko, Mu eki.

6- aparecer: Guarani obapo, Mu jebapik.

7- apoiar: $\mathrm{Tb} k o k,{ }^{*} k o k, \mathrm{Mu} k i k$ cuidar, tratar.

8- aqui: Tb ke, ike, Mu še perto, este invisível.

9- arara: Tb parawa, * Mu i-para a. amarela, ku parawa a. azul.

10- árvore: Tb $2 i b,{ }^{*}$ ?ib, Mu Pip, ku (?)ib.

11- batata doce: $\mathrm{Tb}$ yeṫk, $\mathrm{Mu}$ wešik, ku weǰk.

12- bicho de pé: Tb tun, Mu nõy bicho de pé, Ku noך pulga.

13- cair: $\mathrm{Tb}$ ?ar, * ar, $\mathrm{Mu}{ }^{2} a t, \mathrm{Ku}\left({ }^{2}\right) a d$.

14- caminho: $\mathrm{Tb}$ pe, *pe, $\mathrm{Mu} e, \mathrm{Ku}-\varepsilon$.

15- casa: $\mathrm{Tb} o k,{ }^{*} o k, \mathrm{Mu} i k-{ }^{\circ} a$.

16- casca: $\mathrm{Tb}$ pe, *pe, $\mathrm{Mu} i-b e$.

17- causativo: $\mathrm{Tb} \mathrm{mo}^{-},{ }^{*} \mathrm{mo}-\mathrm{Mu} \mathrm{m}-\mathbf{i}^{-}$.

18- causativo-comitativo: Tb ero-, *ero-, Mu ijit-.

19- cabeça: $\mathrm{Tb}{ }^{\circ} a, \mathrm{Mu}{ }^{\circ} a, \mathrm{Ku}\left({ }^{\circ}\right) a$. 
20- cavar: Tb íbí-kõy, ${ }^{*} \dot{i} b \dot{i}-k \tilde{o} y, \mathrm{Mu}$ kĩy buraco no chão.

21- chegar: Tb w-asem, *w-acem, Mu ajẽm.

22- cipó: Tb isiptpo, Mu išibi, Ku ijibi.

23- cobra: Tb moy, " moy, Mu piy, Ku pëy.

24- cobrir: Tb asoy, *acoy, Mu ajiy coberto.

25- com: Tb ese, *ece, Mu ejt, Ku edi.

26- comer: $\mathrm{Tb}{ }^{~} u$, ${ }^{*} u, \mathrm{Mu}{ }^{\circ} \mathrm{o}, \mathrm{Ku}\left({ }^{\prime}\right) u$ beber.

27- cru: Tb pir, Mu it.

28- cupim: Tb kupi?i, "kupi'i, Mu i-kopi vespa.

29- dativo: $\mathrm{Tb}-b e,{ }^{*}-b e, \mathrm{Mu}-b e, \mathrm{Ku}-b \varepsilon$.

30- de: Tb s-u?i, Guarani -wi, $\mathrm{Mu}-b e-w i, \mathrm{Ku}-b \varepsilon-y$.

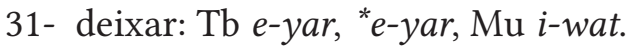

32- dente: Tb $\tilde{a} y,{ }^{*} \tilde{a} y, \mathrm{Mu} \tilde{t} y, \mathrm{Ku} \tilde{a} i$.

33- dizer: $\mathrm{Tb}{ }^{\prime} e,{ }^{*} e, \mathrm{Mu}$ 'e .

34- dormir: $\mathrm{Tb} k e r,{ }^{*} k e r, \mathrm{Mu}$ šet, $\mathrm{Ku}$ šed.

35- dorso: Tb kupe, "kupe, Mu kopi espinha dorso, Ku ku dorso.

36- ele: $\mathrm{Tb} i-,{ }^{*} i-, \mathrm{Mu} i-, \mathrm{Ku} i$-.

37- ele: $\mathrm{Tb} o_{-},{ }^{*} \mathrm{o}^{-}, \mathrm{Mu}{ }^{\circ} \mathrm{o}^{-}, \mathrm{Ku} u$ -

38- ele: $\mathrm{Tb} t-,{ }^{*} t-, \mathrm{Mu} t-, \mathrm{Ku} t$-.

39- ele: $\mathrm{Tb} y o-,{ }^{*} y o-, \mathrm{Mu}$ jo-.

40- em: $\mathrm{Tb}-p e,{ }^{*} p e, \mathrm{Mu}-b e, \mathrm{Ku}-b \varepsilon$.

41- em cima de: $\mathrm{Tb}$ sose, $\mathrm{Mu}$ ǰeje, $\mathrm{Ku} d \varepsilon d \varepsilon$.

42- engolir: Tb mo-kon, Mu je-kõn comer, kõn beber.

43- escarnecer: Tb yay, * ${ }^{*}$ ay, $\mathrm{Mu}$ way rir, Ku oay (/way/) rir.

44- estar de pé: $\mathrm{Tb}$ 'am, *am, Mu im lá bem alto.

45- estar deitado: $\mathrm{Tb} u b$, * $u b, \mathrm{Mu}$ op ficar.

46- face: Tb oba, *oba, Mu opa, Ku upa.

47- fibra: $\mathrm{Tb}$ po, * $p o \mathrm{Mu} b \dot{t}$.

48- fígado: Tb $p i^{3} a,{ }^{*} p \dot{t}^{3} a, \mathrm{Mu} p s a$.

49- filho do homem: $\mathrm{Tb} a^{\text {'tir, }}{ }^{*} a^{\mathrm{\prime}} \mathrm{i} r, \mathrm{Mu}$ 'it filho da mulher.

50- flecha: Tb $u^{\prime} u b$, * $u^{\prime} i b, \mathrm{Mu} o p, \mathrm{Ku} o u b, u i b$.

51- flor: Tb po-tir, " $p o-t i r, \mathrm{Mu}$ tit, $\mathrm{Ku}$ tit.

52- fogo: Tb ata, *ata, Mu aša, Ku aša.

53- folha: $\mathrm{Tb} o b$, *ob, Mu ip, Ku ëib.

54- fonte: Tb yãy, Mu wĩy porto, Ku oaĩ (/wãy/) porto.

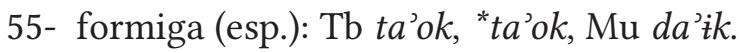

56- fruta: $\mathrm{Tb}{ }^{2} a, \mathrm{Mu}{ }^{2} a, \mathrm{Ku}\left({ }^{\prime}\right) a$.

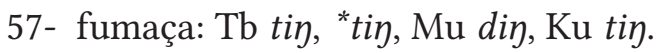

58- fumo: $\mathrm{Tb}$ pe-tim, ${ }^{*} p e-t i m, \mathrm{Mu} e, \mathrm{Ku} e$-.

59- furar: Tb ku-tuk, "ku-tuk, Mu je-tok, mi-tok, Ku natug.

60- gordura: $\mathrm{Tb} k a b,{ }^{*} k a b, \mathrm{Mu}$ šep, $\mathrm{Ku}$ šep-. 


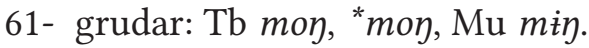

62- imaturo: $\mathrm{Tb} k i r,{ }^{*} k i r, \mathrm{Mu}$ kit verde.

63- ir: $\mathrm{Tb} s o,{ }^{*} c o, \mathrm{Mu} \breve{j} \dot{i} \mathrm{Ku} \breve{j t}, d \dot{t}$.

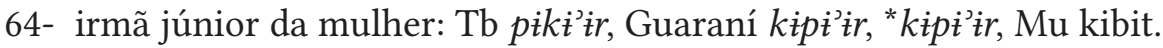

65- irmão da mulher: Tb kibir, *kibir, Mu kipit.

66- irmão júnior do homem: Tb ibirr, ${ }^{*} \dot{b} b \dot{t r}, \mathrm{Ku}$ ipid.

67- jacu: Tb yaku, Mu wakõ, Ku waku.

68- jogar: $\mathrm{Tb} i t i k,{ }^{*} i t i k, \mathrm{Mu}$ šik.

69- lavar: Tb ey, *ey, Mu wiy.

70- língua: Tb ape-kũ, Guaraní kũ, Mu kõ, Ku kũ.

71- lua: Tb yasi, " yaci, Mu kaši, Ku waǰi.

72- mãe: Tb sit, ${ }^{*} c \dot{i}, \mathrm{Mu} \check{s} i, \mathrm{Ku}$ ji.

73- mão: Tb po, *po, Mu bỉ dedo, Ku bi mão.

74- matar: Tb yuka, "yuka, Mu yaoka, Ku yauka-.

75- mato de: $\mathrm{Tb} t \dot{t} b,{ }^{*} t i b, \mathrm{Mu} d i p, \mathrm{Ku} t i b$.

76- mel: Tb eir, *eir, Mu eit, Ku eid.

77- morrer: Tb e’õ , *e $e^{3}, \mathrm{Mu} \tilde{t}^{2}$.

78- mutum: Tb mitũ, " mitũu, Mu wĩtõ, Ku muitũ.

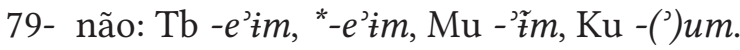

80- nariz, ponta do: $\mathrm{Tb} a p \tilde{t} y, \mathrm{Mu} a b i$ focinho, ponta.

81- nominalizador: $\mathrm{Tb}-a b,{ }^{*}-a b, \mathrm{Mu}-a p, \mathrm{Ku}-a b$.

82- nominalizador: $\mathrm{Tb}-a r,{ }^{*}-a r, \mathrm{Mu}-a t, \mathrm{Ku}-a d$.

83- nós exclusivo: $\mathrm{Tb}$ ore, *ore, $\mathrm{Mu}$ oče, $\mathrm{Ku}$ ute.

84- nós inclusivo: $\mathrm{Tb}$ ya-, $\mathrm{Mu}$ wiy-, $\mathrm{Ku}$ wei-.

85- ovo: Tb upi'a, *upi’a, Mu opsa, Ku upia.

86- paciente: $\mathrm{Tb}-p i r,{ }^{*}-p i r, \mathrm{Mu}-b i t$.

87- pai: $\mathrm{Tb} u b,{ }^{*} u b, \mathrm{Mu}$ op marido, $\mathrm{Ku} u p$ pai, marido.

88- papagaio: Tb ayuru, *ayuru, Mu aro, Ku aru.

89- papai: $\mathrm{Tb} p a^{\jmath} i, \mathrm{Mu}$ bay pai, Ku bai pai.

90- passar: Tb kwab, " $k^{w} a b, \mathrm{Mu} k a p, \mathrm{Ku} k a b$.

91- pau de cavar: Tb sir, Mu šit pá.

92- pé: $\mathrm{Tb} p i,{ }^{*} p i, \mathrm{Mu} i, \mathrm{Ku} i$.

93- pedra: Tb ita, *ita, Mu wita- ${ }^{\circ} a, \mathrm{Ku}$ wita-(')a.

94- pegada: Tb pipor, " pipor, Mu ibit, Ku ibid.

95- pegar: Tb pisik, " picik, Mu išik.

96- peito: Tb kam, *kam, Mu kĩm, Ku kam.

97- pelo: $\mathrm{Tb} a b,{ }^{*} a b, \mathrm{Mu} a p$ cabelo, $\mathrm{Ku} a b$ pelo, cabelo.

98- pena: $\mathrm{Tb} a b,{ }^{*} a b, \mathrm{Mu} a p$.

99- perder-se: Tb opar, *opar, Mu opat perder.

100-pesado: Tb posity, * poci̇y, Mu poši,

101-piolho: Tb kib, "kib, Mu kip, Ku kib. 
102-pintado: Tb parab, * parab, Mu bara-rak, Ku bara-rag.

103-polpa: Tb u’um, Mu -õm.

104-possuidor: Tb yar, " yar, Mu i-wat.

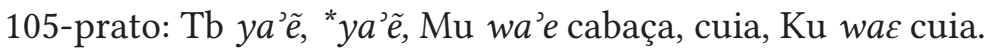

106-preguiça, bicho: $\mathrm{Tb} a^{3} \dot{t},{ }^{*} a^{3} \dot{t}, \mathrm{Mu} a y$.

107-quebrar: Tb $k a,{ }^{*} k a, \mathrm{Mu} k a$.

108-quem: Tb aba, *aba, Mu abi, Mu abui.

109-queimar: Tb a-pi, Mu pik (intr.).

110-quente: Tb akub, *akub, Mu ašip, Ku akib.

111-querer: Tb sey, *cey, Mu jity.

112-rabo: Tb uway, "uway, Mu oay.

113-raiz: $\mathrm{Tb}$ apo, *apo, $\mathrm{Mu}-a b \dot{\text {. }}$.

114-ralar: $\mathrm{Tb} e, \mathrm{Mu} e$.

115-receber: Tb yar, ar, *yar, "ar, Mu jat agarrar.

116-rede: $\mathrm{Tb}$ inĩ, $\mathrm{Ku}$ ini.

117-reflexivo: $\mathrm{Tb} y e-,{ }^{*} y e-, \mathrm{Mu}$ je-we.

118-residente de: $\mathrm{Tb}$-war, ${ }^{*}$-war, $\mathrm{Mu}$-wat.

119-respirar: Tb pitu, "pitu, Mu -pido-, Ku bidu respiração.

120-roça: Tb ko, *ko, Mu ki.

121-sair: Tb sem, " cem, Mu jẽm.

122-sangue: $\mathrm{Tb} u w i,{ }^{*} u w i, \mathrm{Mu} o y, \mathrm{Ku} u i$.

123-sentar: Tb apik, "apik, Mu abik, Ku abig.

124-sol: Tb kwar, * $k^{w} a r, \mathrm{Mu} k a-s ̌ i, \mathrm{Ku} k a-j i$.

125-terra: $\mathrm{Tb} i b \dot{i},{ }^{*} i b i, \mathrm{Mu} i p i, \mathrm{Ku} i p i$.

126-tipóia: Tb tipoy, Mu tobiy.

127-tirar: $\mathrm{Tb}$ 'ok, *'ok, Mu 'itk.

128-tamar banho: Tb yasuk, *yacuk, Mu aǰk, Ku aduk.

129-trançar: $\mathrm{Tb}$ pẽ, * ${ }^{*} \tilde{e}, \mathrm{Mu}$ pen torcer.

130-tu: Tb $e-$, ${ }^{*} e-, \mathrm{Mu} e-, \mathrm{Ku} \varepsilon$ -

131-tu: Tb ene, *ene, Mu ẽn, Ku $\varepsilon n$.

132-tucano: Tb tukan, " tukan, Mu čokõn, Ku tukan.

133-urubu: Tb urubu, *urubu, Mu oropo, Ku urupu.

134-veado: Tb si-wasu, Mu i-ši-, Ku i-ǰi.

135-vento: Tb ibitu, *ibitu, Mu kabido, Ku kabidu.

136-vir: Tb yur, ur, ${ }^{*} y u r,{ }^{*} u r, \mathrm{Mu}$ jot, ot, $\mathrm{Ku} u d$.

137-visitar: Tb sub, *cub, Mu jop ver.

138-voltar: Tb yebir, “yebir, Mu jepit.

139-vós: Tb pe-, " $p e-$, Mu ey-, Ku ei-.

140-calcanhar: Tb pita, " ${ }^{*}$ ita, Mu ida.

141- morrer: $\mathrm{Tb}$ pab, ${ }^{*}$ pab, Mu a-pap morrer muita gente. 


\section{Notas}

(1) Um desenvolvimento relativamente recente em Mundurukú, que não ocorreu em Kuruáya, é pia > psa: 85 - ovo Mu opsa <*opia $=\mathrm{Ku}$ upia $=\mathrm{Tb}$ upia, *upi a; 48 - fígado Mu psa (Ku não documentado) < ${ }^{*} p i a=\mathrm{Tb} p \dot{t}^{3} a,{ }^{*} p \dot{t}^{3} a$.

(2) Para os números 6 e 108 foram postulados, em estudo anterior, ainda inédito, Proto-Tupí * $e p^{w} a p o k^{\prime}$ e * $a p^{w} o$, respectivamente, à vista do Tuparí (fam. Tuparí) epapok chegar e apo quem; proto-Tupí * $p^{w}$ daria em Proto-Tupí-Guaraní (e em Mundurukú) * $b$ (além do arredondamento, em Proto-Tupí-Guaraní, da vogal precedente) *obapo $\left({ }^{*}-k^{\prime}\right.$ daria $-k$ em Tuparí, mas $\emptyset$ em proto-Tupí-Guaraní). O número 29, não ocorrendo em Tuparí, não foi considerado naquele estudo anterior, mas em princípio nada parece opor-se à postulação de Proto-Tupí * $-p^{w} e$. Quanto ao número 135, parece que ambas as formas são compostos haplológicos, incluindo o morfema do número 119: Proto-Tupí-Guaraní * ${ }^{b} b \dot{i}$ (pi) tu respiração da terra (cf. no 125) e Proto-Mundurukú * $k a^{3} b i$ (pi) do respiração do céu $\left(M u k a^{3} b i\right.$ céu); mas fica sem explicação a ausência de ' no $\mathrm{Mu}$ kabido. Uma dificuldade para a hipótese de que a correspondência 4 provém do proto-Tupí * $p$ apresentada, entretanto, pelo número 46, que se encaixa na correspondência 3: o tupari epa olho, luz em confronto com o proto-Tupí-Guaraní * oba rosto, leva à postulação de proto-Tupí * $e p^{w} a$, precisamente como no caso de *obapo < * $e p^{w} a p o k$, mas aqui o Mundurukú tem outro comportamento.

(3) Esta correspondência se prende ao fonema * $t$ ' postulado para o Proto-Tupí com a evidência do Tuparí, que apresenta $h$ em início e $\emptyset$ em meio de morfema: ao número 2 compare-se o Tuparí hap cobertura do teto, kawconstruir; ao número 119, o Tuparí cio respiração; ao número 135, o Tuparí ipcio vento.

(4) Os números 12 e 132 foram associados por corresponderem ao Tuparí yõ-tap bicho de pé e yõkan tucano, respectivamente. $\mathrm{O}$ fonema tentativamente proposto para o Proto-Tupí é * c': * c'u⿰ e *ç'ukan.

(5) Os números 18 e 83, em que $\mathrm{Tb} r,{ }^{*} r$ correspondem a uma obstruinte palatal em $\mathrm{Mu}$, têm ambos contrapartes com t em Tuparí: ete- e ote, respectivamente.

(6) O número 110 é um caso à parte, pois embora o $\mathrm{Mu}$ apresente š, o Ku não o acompanha como nos demais casos. Evidentemente a forma ProtoMundurukú deve ter sido análoga à do Kuruáya, *akip, e a mudança de $k$ em š no Mundurukú talvez seja um fenômeno analógico possivelmente devido à estreita associação semântica com Mu a ša fogo.

(7) Nimuendajú (1930) sistematicamente deixa de registrar as oclusivas glotais, que provavelmente ocorriam em Kuruáya tanto quanto em Mundurukú. 
(8) No número 88 o Mu aro deve provir de *aworo (cf. Tuparí aoro papagaio), com ${ }^{*} w>\emptyset$. Também ${ }^{*} w>\emptyset$ no número 76 , cuja reconstrução Proto-Tupí deve ser * ewir (cf. Tuparí ewit); em Proto-Tupí-Guaraní * $y>\emptyset$ diante de * $i$, não só nesse caso, mas também no número 93, cuja forma Proto-Tupí seria * wita.

(9) Há um único caso, o número 118, de w em Tupinambá e Mundurukú; é possível que se trate de empréstimo no Mundurukú, tomado a uma língua Tupí-Guaraní, como p. ex. a Língua Geral (Nheengatú).

(10) $\mathrm{Mu} / \mathrm{u}$ varia foneticamente de $[\mathrm{o}]$ a $[\mathrm{u}]$; margem de variação análoga apresenta $\mathrm{Mu} / \tilde{\mathrm{u}} /$.

(11) No número 106, Mu ay pressupõe os intermediários * $a^{3} i \mathrm{e}$ * $a i$ (cf. correspondência 12 e nota 12 )

(12) No número 89 o $i$ fica contíguo ao a após a queda de ' (correspondência 12) e torna-se assilábico, como no número 106 (cf. nota 11).

(13) O número 42 apresenta $o=$ õ em sílaba acentuada; é provável que se trate de morfemas fonologicamente parecidos, mas não cognatos.

(14) Os números 84 e 108 apresentam $a=\dot{t}$. No caso do número 108 há evidência clara de que o $\mathrm{Mu} \dot{i}$ corresponde realmente ao Proto-Tupí * $o$, conforme o testemunho do Tuparí apo quem (cf. nota 2); o - $a$ do Proto-Tupí-Guaraní * $a b a$ se deve, provavelmente, a assimilação: Proto-Tupí * $a p^{w_{0}}>$ > Proto-TupíGuaraní *abo > *aba, analogamente a Proto-Tupí-Guaraní *aco > *aca eu fui, *yaco > *yaca nós fomos (cf. Guaraní aha, yaha). Quanto ao número 84, a situação não é tão clara; mas $o k u$ wei- parece indicar que o $\dot{i}$ do $\mathrm{Mu}$ wiy- provém imediatamente de um $e$, como na correspondência 24, e não de um $a$; neste caso, o Proto-Mundurukú teria * wey- correspondendo ao Proto-Tupí-Guaraní * $y a-$; o $-y$ da forma Proto-Mundurukú parece ser um formante comum aos marcadores de $1^{\text {a }}$, pessoa inclusiva e $2^{\text {a }}$ pessoa do plural: $\mathrm{cp}$. $\mathrm{Mu}$ ey-, $\mathrm{Ku}$ ei- = $\mathrm{Tb}$ pe- (número 139); a correspondência restante, $\mathrm{Tb} a,{ }^{*} a=\mathrm{Mu} e, \mathrm{Ku} e$ tem paralelo no número $60, \mathrm{~Tb} k a b$, ${ }^{*} k a b=\mathrm{Mu}$ šep, $\mathrm{Ku}$ šep-, e deve-se, possivelmente, a uma vogal Proto-Tupí distinta de * $a$.

(15) Os números 18 e 25 apresentam também $e=i$, mas em contextos em que normalmente ocorre $e=e$. Em 18 é possível que, no Mundurukú, $\dot{t}$ resulte

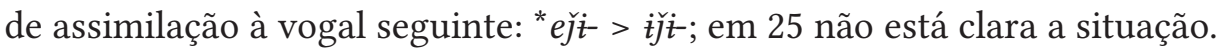

(16) Um caso que escapa nitidamente ao padrão desta correspondência é o número 57, que apresenta vogal em Mundurukú.

\section{Bibliografia}

Anônimo. 1952-1953. Vocabulário na língua brasílica. $2^{\mathrm{a}}$ edição por Carlos Drumond. 2 volumes; São Paulo; Universidade de São Paulo.

Braun, Ilse, e Marjorie Crofts. 1965. "Munduruku Phonology”. Anthropogical Linguistics 7:7:23- 39. 
Crofts, Marjorie. 1973. Gramática Mundurukú. Brasília. Summer Institute of Linguistics.

Lemle, Miriam. 1971. "Internal Classification of the Tupí-Guaraní Linguistic Family”. Tupi Studies I (David Bendor-Samuel, ed.): 107-129. Norman: Summer Institute of Linguistics.

Nimuendajú, Curt. 1930. "Zur Sprache der Kuruáya-Indianer". fournal de la Société des Américanistes 22:317-345.

Nimuendajú, Curt. 1937. "Die Verwandtschaft des Mundurukuischen mit dem Tupíischen". Santo Antonio, Provinzzeitschrift der Franziskaner in Nordbrasilien 15: 2:76-80.

Rodrigues, Aryon D. 1955. "As línguas "impuras” da família Tupí-Guarani”". Anais do XXXI Congresso Internacional de Americanistas: 1055-1071. São Paulo.

Rodrigues, Aryon D. 1958. Phonlogie der Tupinambá-Sprache. Dissertation, Universität Hamburg.

Rodrigues, Aryon D. 1970. "Línguas ameríndias". Grande Enciclopédia Delta-Larousse: 4034-4036. Rio de Janeiro: Delta.

Ruiz de Montoya, Antonio. 1876. Vocabulario y tesoro de la lengua guaraní, ó más bien tupi. Nueva edición. Viena e Paris.

Strömer, Chrisostamus. 1932. Die Sprache der Munduruku. Mödling bei Wien: Anthropos. 